\title{
Using Big Healthcare Data to Supplement Chikungunya Surveillance in the U.S.
}

\author{
Joel Greenspan*1 and Silvia Valkova ${ }^{2}$ \\ 'Martin, Blanck \& Associates, Atlanta, GA, USA; ${ }^{2}$ IMS Health Government Solutions, Fairfax, VA, USA
}

\section{Objective}

This paper describes how high-volume electronic healthcare (HC) reimbursement claims (eHRCs) from providers' offices can be used to supplement Chikungunya surveillance in the U.S.

\section{Introduction}

Chikungunya virus disease (CHIK) is a mosquito-borne viral infection currently widespread in the Caribbean with the potential for emergence and endemicity in the U.S. via infected travelers and local mosquito vectors. CHIK disease can be severe and disabling with symptoms similar to dengue. CHIK is not a U.S. nationally notifiable disease and tracking travel-associated and locally acquired cases is currently dependent on voluntary reporting via ArboNET. While ArboNET cases are laboratory confirmed and highly specific, ArboNET is a passive surveillance system where representativeness and timeliness may be lacking. In contrast, submitting an electronic bill following $\mathrm{HC}$ services is the most mature and widely used form of eHealth. Providers are highly motivated to submit claims for reimbursement and the eHRC process is ubiquitous in the U.S. HC system. HIPAA-compliant eHRCs from provider offices can be captured in e-commerce and consolidated into electronic data warehouses and used for many purposes including public health surveillance. eHRCs are standardized and each claim contains pertinent person, place, and time information as well as ICD-9 diagnostic codes. IMS Health (IMS) is a global HC information company and maintains one of world's largest eHealth data warehouses that processes $\sim 1$ billion provider office eHRCs annually. IMS consolidates eHRCs from $>60 \%$ of all U.S. office-based providers from all parts of the U.S. The size and predictability of the eHRC flow into the IMS data warehouse supports projections of national estimates and time trends of conditions of interest.

\section{Methods}

For this study we compared CHIK cases reported via ArboNET from January to August 2014 with eHRC provider claims with an ICD-9 code of 066.3 (Other Mosquito-borne Fever) that had been received in the IMS data warehouse during the same time period. We classified 066.3 patients as suspect CHIK cases. eHRC data elements examined included unique patient ID, month of provider visit, and patient State and ZIP-3. For patients with multiple provider 066.3 visits, the earliest visit was used for analysis.

\section{Results}

During the observation period ArboNET reported 584 CHIK cases from 42 States and D.C. IMS noted 509 suspect cases from 40 states. The distribution of States with any CHIK cases was similar between ArboNet and IMS. However, ArboNET reported cases in 5 States where IMS detected none; IMS detected suspect cases in 2 states where ArboNET reported none. ArboNET reported many more cases in 2 States (FL, TN); IMS reported many more suspect cases in 5 States (IL, MI, NY, PA, WV). The State with the most ArboNET cases was FL (142); the State with the most IMS suspect cases was NY (125). IMS ZIP-3 analysis demonstrated a high proportion of suspect cases in several high density urban areas (NYC metro and
Northern NJ, Philadelphia, Detroit). IMS suspect cases in FL were noted in Miami and other major population areas.

\section{Conclusions}

eHRCs may help overcome some of the limitations of ArboNET in documenting timing, occurrence, and spread of CHIK in the U.S., but not without additional research to verify the predictive value of ICD-9 066.3 and whether providers are using this code based on known laboratory values or general awareness of the disease, its symptoms, and patient recent travel history.

\section{Keywords}

Chikungunya; ArboNet; healthcare claims; big data

\author{
*Joel Greenspan \\ E-mail: greenspan@comcast.net
}

